\title{
Chaotic scattering with direct processes: A generalization of Poisson's kernel for non-unitary scattering matrices
}

\author{
V. A. Gopar ${ }^{1,2}$, M. Martínez-Mares ${ }^{3}$, and R. A. \\ Méndez-Sánchez ${ }^{4}$ \\ ${ }^{1}$ Departamento de Física Teórica, Facultad de Ciencias, Universidad de Zaragoza, \\ Pedro Cerbuna 12, 50009 Zaragoza, Spain. \\ ${ }^{2}$ Instituto de Biocomputación y Física de los Sistemas Complejos, Universidad de \\ Zaragoza, Corona de Aragón, 42, 50009 Zaragoza, Spain. \\ ${ }^{3}$ Departamento de Física, Universidad Autónoma Metropolitana-Iztapalapa, A. P. \\ 55-534, 09340 México D. F., Mexico. \\ ${ }^{4}$ Instituto de Ciencias Físicas, Universidad Nacional Autónoma de México, A.P. \\ 48-3, 62210, Cuernavaca, Morelos, Mexico.
}

\begin{abstract}
.
The problem of chaotic scattering in the presence of direct processes or prompt responses is mapped via a transformation to the case of scattering in the absence of such processes for non-unitary scattering matrices $\tilde{S}$. When prompt responses are absent, $\tilde{S}$ is uniformly distributed according to its invariant measure in the space of $\tilde{S}$ matrices with zero average $\langle\tilde{S}\rangle=0$. When direct processes occur, the distribution of $\tilde{S}$ is non-uniform and is characterised by an average $\langle\tilde{S}\rangle \neq 0$. In contrast to the case of unitary matrices $S$, where the invariant measures of $S$ for chaotic scattering with and without direct processes are related through the well-known Poisson kernel, we show that for non-unitary scattering matrices the invariant measures are related by the Poisson kernel squared. Our results are relevant to situations where flux conservation is not satisfied, for transport experiments in chaotic systems where gains or losses are present, for example in microwave chaotic cavities or graphs, and acoustic or elastic resonators.
\end{abstract}

PACS numbers: 73.23.-b, 03.65.Nk, 42.25.Bs 


\section{Introduction}

The statistical properties of ensembles of scattering matrices have been studied extensively since their introduction in pioneering works in the field of nuclear physics [1, 2]. More recently, Random Matrix Theory (RMT) techniques have been applied to study several statistical properties of electronic transport in mesoscopic systems [3, 4, 5]. Although the spectrum of applications of RMT is quite wide, ranging from atomic nuclei to microwave cavities, most of the investigations in RMT have considered systems in which flux is conserved. Therefore, the associated $n \times n$ scattering matrix $S$ is unitary,

$$
S S^{\dagger}=I_{n},
$$

where $I_{n}$ denotes the unit matrix of dimension $n$, and the dagger means Hermitian conjugation. Here $n$ denotes the number of scattering channels.

When unitarity is the only constraint, the unitary case denoted by $\beta=2$ in Dyson's scheme applies [6]. If in addition time-reversal symmetry is imposed, then $S$ is also a symmetric matrix,

$$
S=S^{T}
$$

a case corresponding to $\beta=1$. Here, the superscript $T$ denotes transposition. When time-reversal symmetry is present, but spin-rotation symmetry is broken, the $S$ matrix is unitary and self-dual, a case denoted by $\beta=4$.

Any scattering matrix $S$ can be decomposed as

$$
S=U V,
$$

where $U$ and $V$ are $n \times n$ unitary matrices for $\beta=2$, while $V=U^{T}$ for $\beta=1$. For $\beta=4, V=U^{R}$, where $U^{R}$ is the dual matrix of $U$.

It has been shown [3, 4] that chaotic scattering in the absence of direct processes is well described by uniformly distributed $S$-matrices. The uniform distribution is given by the invariant measure defined through the relation

$$
\mathrm{d} \mu_{\beta}(S)=\mathrm{d} \mu_{\beta}\left(U^{\prime} S V^{\prime}\right)
$$

which is assumed to be normalized, i.e.,

$$
\int \mathrm{d} \mu_{\beta}(S)=1
$$

The matrices $U^{\prime}$ and $V^{\prime}$ are arbitrary but fixed $n \times n$ unitary matrices for $\beta=2$, $V^{\prime}=U^{\prime T}$ for $\beta=1$, and $V^{\prime}=U^{\prime R}$ for $\beta=4$. Equation (44) defines the Circular Ensembles, Orthogonal $(\beta=1)$, Unitary $(\beta=2)$, and Symplectic $(\beta=4)$. For these ensembles the average of the scattering matrix satisfies $\langle S\rangle=0$.

When direct processes due to short trajectories exist (prompt responses), the $S$ matrix is no longer uniformly distributed. It turns out that in this case the direct processes can be characterized by the average $\langle S\rangle(\neq 0)$, known as the optical $S$-matrix. In fact, in the maximum-entropy approach developed in the past [3, 7, 8], the probability distribution of $S$ is given by

$$
\mathrm{d} P_{\langle S\rangle}^{(\beta)}(S)=p_{\langle S\rangle}^{(\beta)}(S) \mathrm{d} \mu_{\beta}(S),
$$


where

$$
p_{\langle S\rangle}^{(\beta)}(S)=\frac{\left[\operatorname{det}\left(I_{n}-\langle S\rangle\langle S\rangle^{\dagger}\right)\right]^{(\beta n+2-\beta) / 2}}{\left|\operatorname{det}\left(I_{n}-S\langle S\rangle^{\dagger}\right)\right|^{(\beta n+2-\beta)}}
$$

is the so-called Poisson kernel. We note that for $\langle S\rangle=0$ the Poisson kernel reduces to unity. Therefore, it is clear that the presence of prompt scattering processes makes the analysis of the statistical properties of $S$ more difficult. Fortunately, a scattering matrix which satisfies the Poisson distribution, Equations ( $6 a b)$ and (6b), can be transformed into a matrix $S_{0}$ with uniform distribution by an ad hoc transformation which maps the problem with presence of direct processes into one without such processes, namely

$$
S_{0}=\frac{1}{t_{c}^{\prime}}(S-\langle S\rangle) \frac{1}{I_{n}-\langle S\rangle^{\dagger} S} t_{c}^{\dagger}
$$

where $t_{c}$ and $t_{c}^{\prime}$ satisfy [10, 11]

$$
\begin{aligned}
& t_{c}^{\dagger} t_{c}=I_{n}-\langle S\rangle^{\dagger}\langle S\rangle, \\
& t_{c}^{\prime} t_{c}^{\prime \dagger}=I_{n}-\langle S\rangle\langle S\rangle^{\dagger} .
\end{aligned}
$$

It has been shown that for one-energy scattering matrices, the Jacobian $J_{\beta}$ of the transformation given in (7) is just the Poisson kernel, (하) [3, 8, 9, 10], i.e.,

$$
\mathrm{d} \mu_{\beta}\left(S_{0}\right)=J_{\beta} \mathrm{d} \mu_{\beta}(S) \equiv \frac{\left[\operatorname{det}\left(I_{n}-\langle S\rangle\langle S\rangle^{\dagger}\right)\right]^{(\beta n+2-\beta) / 2}}{\left|\operatorname{det}\left(I_{n}-S\langle S\rangle^{\dagger}\right)\right|^{\beta n+2-\beta}} \mathrm{d} \mu_{\beta}(S) .
$$

The transformation (7) was also used in Ref. [12] to show that the statistical properties of the transformed unitary scattering matrices at several energies $S_{0}\left(E_{1}\right), S_{0}\left(E_{2}\right)$, . are the ones associated to the problem of scattering in the absence of direct processes.

We note from (7) that for $\langle S\rangle=0, S$ reduces to $S_{0}$ and a uniform distribution is recovered for $S$, i.e., $\mathrm{d} P_{\langle S\rangle}^{(\beta)}(S)$ reduces to the invariant measure $\mathrm{d} \mu_{\beta}(S)$. Thus, (9) implies that if $S_{0}$ is uniformly distributed, then the matrix $S$ obtained from (7) is distributed according to the Poisson kernel.

Although the Poisson kernel distribution has been successfully verified, for example in describing several transport properties in quantum dots [3, 4, 10, 13, 14, 15, 16] and disordered waveguides [17], there are situations where flux conservation is violated. Therefore, the corresponding $S$ matrix becomes non-unitary, i.e., (11) is not satisfied. For instance, power losses (absorption) are unavoidable in experiments on microwave cavities and graphs [18, 19, 20], acoustic resonators [21], and elastic media 22]; in those cases the $S$ matrix is sub-unitary. Also, systems with gains (amplification) exist [23], where $S$ is a supra-unitary matrix.

Recently, several efforts have been made in order to incorporate the information of losses or gains (for a review see [24] and [25]; see also [23, 26, 27, 28, 29, 30]). However, few investigations have considered non-unitary scattering matrices $\tilde{S}$ in the presence of direct processes. For example, sub-unitary scattering matrices have been considered in Refs. [31, 32, 33]. There, the prompt responses come from direct reflections due to an imperfect coupling of the antenna to the cavity and the optical scattering matrix $\langle\tilde{S}\rangle$ 
was assumed to be a diagonal matrix. In what follows we will consider the general case when $\langle\tilde{S}\rangle$ is a full matrix.

With the same philosophy of Ref. [8], in this paper we transform a non-unitary scattering matrix $\tilde{S}$, with $\langle\tilde{S}\rangle \neq 0$, into a non-unitary scattering matrix $\tilde{S}_{0}$ for which $\left\langle\tilde{S}_{0}\right\rangle=0$, by using the transformation (77). We show that the Jacobian $\tilde{J}_{\beta}$ associated to this transformation is given by the square of the Poisson kernel, (6b) with $S$ replaced by $\tilde{S}$. We consider the cases of $n \times n$ non-unitary scattering matrices in the presence $(\beta=1)$ and absence $(\beta=2)$ of time reversal symmetry. Self dual $\tilde{S}$-matrices $(\beta=4)$ are also studied.

The paper is organized as follows. The next section is devoted to the invariant measure for non-unitary matrices. In Sect. 3 we write $\tilde{S}_{0}$ in terms of $\tilde{S}$ using the transformation in (7) and the corresponding Jacobian $\tilde{J}_{\beta}$ is calculated. The one-channel case is presented as a simple example in Sect. 4. Finally, we present the conclusions in Sect. [5,

\section{The invariant measure for non-unitary matrices}

Let $\tilde{S}$ be an $n \times n$ non-unitary scattering matrix,

$$
\tilde{S} \tilde{S}^{\dagger} \neq I_{n}
$$

As for the unitary scattering matrices, we consider the following cases. In the presence of time-reversal invariance $\tilde{S}=\tilde{S}^{T}$ and we will refer to this case as $\beta=1$; in the absence of time-reversal invariance $\tilde{S}$ has no restriction, and we denote this case by $\beta=2$. Finally, when $\tilde{S}=\tilde{S}^{R}, \beta=4$.

As in (3), any non-unitary matrix $\tilde{S}$ can be parametrized as $[9,33,34$,

$$
\tilde{S}=U \rho V,
$$

where $\boldsymbol{\rho}$ is a real diagonal matrix whose diagonal elements are positive, $\boldsymbol{\rho}_{a b}=\rho_{a} \delta_{a b}$. For sub-unitary matrices the eigenvalues of $\tilde{S} \tilde{S}^{\dagger}$ are real numbers between 0 and 1 , while they are larger than 1 for supra-unitary matrices. $U$ and $V$ are defined in the same way than in (3) for each $\beta$ symmetry. Note that for $\rho_{a}=1, \tilde{S}$ reduces to a unitary $S$-matrix and $\tilde{S}$ vanishes for $\rho_{a}=0$. In this sense, the diagonal elements $\rho_{a}$ determine the strength of the absorption or amplification [33].

The uniform distribution for an ensemble of $\tilde{S}$-matrices is given by the invariant measure, defined as in (4), by replacing $S$ by $\tilde{S}$. In terms of the independent elements of $\tilde{S}, \mathrm{~d} \mu_{\beta}(\tilde{S})$ is given by [2, 8]

$$
\mathrm{d} \mu_{\beta}(\tilde{S})=\prod_{\{a, b\}} \mathrm{d}\left(\operatorname{Re} \tilde{S}_{a b}\right) \mathrm{d}\left(\operatorname{Im} \tilde{S}_{a b}\right), \quad \text { for } \beta=1,2 .
$$

We have used the following notation for the indices for symmetric complex $(\beta=1)$ and complex $(\beta=2) \tilde{S}$-matrices:

$$
\{a, b\}= \begin{cases}a \leq b=1, \ldots, n & \text { for } \beta=1, \\ a, b=1, \ldots, n & \text { for } \beta=2 .\end{cases}
$$


For self-dual $\tilde{S}$-matrices $(\beta=4)$ with complex quaternion components $\tilde{S}_{a b}^{(\alpha)}$ we have

$$
\mathrm{d} \mu_{4}(\tilde{S})=\prod_{a<b} \prod_{\alpha=1}^{3} \mathrm{~d}\left(\operatorname{Re} \tilde{S}_{a b}^{(\alpha)}\right) \mathrm{d}\left(\operatorname{Im} \tilde{S}_{a b}^{(\alpha)}\right) \prod_{a \leq b} \mathrm{~d}\left(\operatorname{Re} \tilde{S}_{a b}^{(0)}\right) \mathrm{d}\left(\operatorname{Im} \tilde{S}_{a b}^{(0)}\right) .
$$

Equations (12) and (14) ensure that $\langle\tilde{S}\rangle=0$, as it should be for a uniform distribution of $\tilde{S}$.

By differentiation of (11) we obtain

$$
\mathrm{d} \tilde{S}=\left(U \boldsymbol{\rho}^{1 / 2}\right) \delta M\left(\boldsymbol{\rho}^{1 / 2} V\right),
$$

where

$$
\delta M=\boldsymbol{\rho}^{-1 / 2} U^{\dagger} d U \boldsymbol{\rho}^{1 / 2}+\boldsymbol{\rho}^{-1 / 2} d \boldsymbol{\rho} \boldsymbol{\rho}^{-1 / 2}+\boldsymbol{\rho}^{1 / 2} d V V^{\dagger} \boldsymbol{\rho}^{-1 / 2} .
$$

We note that $\delta M$ is in general a complex matrix $(\beta=2)$; in addition, it is symmetric for $\beta=1$ and self-dual for $\beta=4$. Alternatively, the invariant measure can be written as

$$
\mathrm{d} \mu_{\beta}(\tilde{S})=\prod_{\{a, b\}} \operatorname{Re}\left(\delta M_{a b}\right) \operatorname{Im}\left(\delta M_{a b}\right), \quad \text { for } \beta=1,2,
$$

and, for $\beta=4$,

$$
\mathrm{d} \mu_{4}(\tilde{S})=\prod_{a<b} \prod_{\alpha=1}^{3} \operatorname{Re}\left(\delta M_{a b}^{(\alpha)}\right) \operatorname{Im}\left(\delta M_{a b}^{(\alpha)}\right) \prod_{a \leq b} \operatorname{Re}\left(\delta M_{a b}^{(0)}\right) \operatorname{Im}\left(\delta M_{a b}^{(0)}\right),
$$

where $\delta M_{a b}^{(\alpha)}$ is the $\alpha$-th quaternion component of $\delta M_{a b}$ (see Appendix A).

\section{Mapping the scattering problem from presence to absence of direct processes}

Let us consider an ensemble of non-uniformly distributed $\tilde{S}$ matrices with average $\langle\tilde{S}\rangle \neq 0$. We now apply the transformation given by (17) to $\tilde{S}$ matrices in order to obtain an ensemble of $\tilde{S}_{0}$ matrices with uniform distribution, i.e.,

$$
\tilde{S}_{0}=\frac{1}{\tilde{t}_{c}^{\prime}}(\tilde{S}-\langle\tilde{S}\rangle) \frac{1}{I_{n}-\langle\tilde{S}\rangle^{\dagger} \tilde{S}} \tilde{t}_{c}^{\dagger}
$$

where $\tilde{t}_{c}$ and $\tilde{t}_{c}^{\prime}$ satisfy (similarly to Equations $(\underline{8 a})$ and $(\underline{8 b})$ )

$$
\begin{aligned}
& \tilde{t}_{c}^{\dagger} \tilde{t}_{c}=I_{n}-\langle\tilde{S}\rangle^{\dagger}\langle\tilde{S}\rangle \\
& \tilde{t}_{c}^{\prime} \tilde{t}_{c}^{\prime \dagger}=I_{n}-\langle\tilde{S}\rangle\langle\tilde{S}\rangle^{\dagger}
\end{aligned}
$$

Then we look for the Jacobian $\tilde{J}_{\beta}$ of this transformation.

By direct differentiation of $\tilde{S}_{0}$ we obtain

$$
\mathrm{d} \tilde{S}_{0}=A^{\prime} \mathrm{d} \tilde{S} A,
$$

with $A$ and $A^{\prime}$ the complex matrices

$$
\begin{aligned}
& A=\left(I_{n}-\langle\tilde{S}\rangle^{\dagger} \tilde{S}\right)^{-1} \tilde{t}_{c}^{\dagger}, \\
& A^{\prime}={\tilde{t^{\prime}}}_{c}^{\dagger}\left(I_{n}-\tilde{S}\langle\tilde{S}\rangle^{\dagger}\right)^{-1},
\end{aligned}
$$


for $\beta=2 . A^{\prime}=A^{T}$ for $\beta=1$ and $A^{\prime}=A^{R}$ for $\beta=4$. On the other hand, a similar expression to (15) is valid for $\tilde{S}_{0}$ as well, namely

$$
\mathrm{d} \tilde{S}_{0}=\left(U_{0} \boldsymbol{\rho}_{0}^{1 / 2}\right) \delta M_{0}\left(\boldsymbol{\rho}_{0}^{1 / 2} V_{0}\right) .
$$

Substituting Equations (15) and (23) into (21), we obtain the relation between the matrices $\delta M_{0}$ and $\delta M$,

$$
\delta M_{0}=B^{\prime} \delta M B
$$

where $\delta M_{0}$ has the same structure of (16) and

$$
\begin{aligned}
& B=\boldsymbol{\rho}^{1 / 2} V A V_{0}^{-1} \boldsymbol{\rho}_{0}^{-1 / 2}, \\
& B^{\prime}=\boldsymbol{\rho}_{0}^{-1 / 2} U_{0}^{-1} A^{\prime} U \boldsymbol{\rho}^{1 / 2},
\end{aligned}
$$

for $\beta=2$. Again, $B^{\prime}=B^{T}$ for $\beta=1$ and $B^{\prime}=B^{R}$ for $\beta=4$. We remark that $\delta M_{0}$ and $\delta M$ are complex matrices, hence $B$ and $B^{\prime}$ are also complex, in contrast to the case of unitary scattering matrices where $\delta M_{0}$ and $\delta M$ may be taken as real matrices. In the following we will restrict ourselves to the cases $\beta=1$ and 2 , unless explicitly indicated otherwise. The case $\beta=4$ is considered in Appendix A.

It is convenient to separate the real and imaginary parts of $\delta M_{0}$ and $\delta M$ to obtain the Jacobian of the transformation as in (17),

$$
\begin{aligned}
& \operatorname{Re}\left(\delta M_{0 a b}\right)=\sum_{c, d=1}^{n} \operatorname{Re}\left(B_{a c}^{\prime} B_{d b}\right) \operatorname{Re}\left(\delta M_{c d}\right)+\sum_{c, d=1}^{n} \operatorname{Re}\left(\mathrm{i} B_{a c}^{\prime} B_{d b}\right) \operatorname{Im}\left(\delta M_{c d}\right), \\
& \operatorname{Im}\left(\delta M_{0 a b}\right)=\sum_{c, d=1}^{n} \operatorname{Im}\left(B_{a c}^{\prime} B_{d b}\right) \operatorname{Re}\left(\delta M_{c d}\right)+\sum_{c, d=1}^{n} \operatorname{Im}\left(\mathrm{i} B_{a c}^{\prime} B_{d b}\right) \operatorname{Im}\left(\delta M_{c d}\right) .
\end{aligned}
$$

Next, we calculate the Jacobian $\tilde{J}_{a b}^{(\beta)}$ of the transformation which relates the real and imaginary parts of the independent elements of $\delta M_{0}$ with those of $\delta M$ as

$$
\operatorname{Re}\left(\delta M_{0 a b}\right) \operatorname{Im}\left(\delta M_{0 a b}\right)=\tilde{J}_{a b}^{(\beta)} \operatorname{Re}\left(\delta M_{a b}\right) \operatorname{Im}\left(\delta M_{a b}\right) .
$$

To this end, and following Ref. [8], we start by assuming that $B$ and $B^{\prime}$ are diagonal matrices, before considering the general case of any complex matrix.

\subsection{A simple example: $B$ and $B^{\prime}$ diagonal matrices}

Let $B$ and $B^{\prime}$

$$
\begin{aligned}
& B_{a b}=\lambda_{a} \delta_{a b}, \\
& B_{a b}^{\prime}=\lambda_{a}^{\prime} \delta_{a b},
\end{aligned}
$$

where $\lambda_{a}$ 's and $\lambda_{a}^{\prime}$ 's are complex numbers. Therefore (26a) and (26b) reduce to

$$
\begin{aligned}
& \operatorname{Re}\left(\delta M_{0 a b}\right)=\operatorname{Re}\left(\lambda_{a}^{\prime} \lambda_{b}\right) \operatorname{Re}\left(\delta M_{a b}\right)-\operatorname{Im}\left(\lambda_{a}^{\prime} \lambda_{b}\right) \operatorname{Im}\left(\delta M_{a b}\right), \\
& \operatorname{Im}\left(\delta M_{0 a b}\right)=\operatorname{Im}\left(\lambda_{a}^{\prime} \lambda_{b}\right) \operatorname{Re}\left(\delta M_{a b}\right)+\operatorname{Re}\left(\lambda_{a}^{\prime} \lambda_{b}\right) \operatorname{Im}\left(\delta M_{a b}\right) .
\end{aligned}
$$

From these two equations, the Jacobian $\tilde{J}_{a b}^{(\beta)}$ is given by

$$
\tilde{J}_{a b}^{(\beta)}=\left[\operatorname{Re}\left(\lambda_{a}^{\prime} \lambda_{b}\right)\right]^{2}+\left[\operatorname{Im}\left(\lambda_{a}^{\prime} \lambda_{b}\right)\right]^{2}=\left|\lambda_{a}^{\prime} \lambda_{b}\right|^{2} .
$$




\subsection{The general case}

Any complex matrices $B$ and $B^{\prime}$ can be written as

$$
\begin{aligned}
& B=Q \Lambda O, \\
& B^{\prime}=Q^{\prime} \Lambda^{\prime} O^{\prime},
\end{aligned}
$$

where $Q, Q^{\prime}, O, O^{\prime}$ are the most general unitary matrices; we recall that for $\beta=1$, $B^{\prime}=B^{T}$, hence $Q^{\prime}=O^{T}$ and $O^{\prime}=Q^{T}$. Here, $\Lambda$ and $\Lambda^{\prime}$ are diagonal matrices whose diagonal elements $\lambda_{a}$ and $\lambda_{a}^{\prime}$ are complex numbers. We substitute (31a) and (31b) into (24) to obtain

$$
\delta M_{0}=Q^{\prime} \delta M_{2} O
$$

where we have defined

$$
\begin{aligned}
& \delta M_{2}=\Lambda^{\prime} \delta M_{1} \Lambda, \\
& \delta M_{1}=O^{\prime} \delta M Q .
\end{aligned}
$$

We now calculate the Jacobian of the transformation $\delta M_{1} \rightarrow \delta M_{2}$ given by (33). As before, it is convenient to separate the real and imaginary parts,

$$
\begin{aligned}
& \operatorname{Re}\left(\delta M_{2 a b}\right)=\operatorname{Re}\left(\lambda_{a}^{\prime} \lambda_{b}\right) \operatorname{Re}\left(\delta M_{1 a b}\right)-\operatorname{Im}\left(\lambda_{a}^{\prime} \lambda_{b}\right) \operatorname{Im}\left(\delta M_{1 a b}\right), \\
& \operatorname{Im}\left(\delta M_{2 a b}\right)=\operatorname{Im}\left(\lambda_{a}^{\prime} \lambda_{b}\right) \operatorname{Re}\left(\delta M_{1 a b}\right)+\operatorname{Re}\left(\lambda_{a}^{\prime} \lambda_{b}\right) \operatorname{Im}\left(\delta M_{1 a b}\right) .
\end{aligned}
$$

This is exactly the same transformation as in $(29 a)$ and $(29 b)$ for the case of diagonal matrices $B$ and $B^{\prime}$. Then, the corresponding Jacobian of the transformation in (35al) and (35b) is $\tilde{J}_{a b}^{(\beta)}$ given by (30), from which we obtain

$$
\prod_{\{a, b\}} \operatorname{Re}\left(\delta M_{2 a b}\right) \operatorname{Im}\left(\delta M_{2 a b}\right)=\tilde{J}_{\beta} \prod_{\{a, b\}} \operatorname{Re}\left(\delta M_{1 a b}\right) \operatorname{Im}\left(\delta M_{1 a b}\right)
$$

where

$$
\tilde{J}_{\beta}=\prod_{\{a, b\}} \tilde{J}_{a b}^{(\beta)}=\left|\prod_{\{a, b\}} \lambda_{a}^{\prime} \lambda_{b}\right|^{2} .
$$

In Appendix B we show the explicit calculation of $\tilde{J}_{\beta}$. We note, on the other hand, that the transformation $\delta M \rightarrow Q^{\prime} \delta M O$ does not change the measure [2]; therefore, from (32) and (34), $\delta M_{0}$ has the same measure as $\delta M_{2}$ while $\delta M_{1}$ has the same measure as $\delta M$. Thus, by transitivity,

$$
\prod_{\{a, b\}} \operatorname{Re}\left(\delta M_{0 a b}\right) \operatorname{Im}\left(\delta M_{0 a b}\right)=\tilde{J}_{\beta} \prod_{\{a, b\}} \operatorname{Re}\left(\delta M_{a b}\right) \operatorname{Im}\left(\delta M_{a b}\right)
$$

which means that the Jacobian $\tilde{J}_{\beta}$ of the transformation given in (19) relates the invariant measures $d \mu\left(\tilde{S}_{0}\right)$ and $d \mu(\tilde{S})$. Inserting the last result in (17) we obtain

$$
d \mu\left(\tilde{S}_{0}\right)=\tilde{J}_{\beta} d \mu(\tilde{S}) .
$$

From (37) for $\beta=1$ and 2 , and (A.12) for $\beta=4, \tilde{J}_{\beta}$ can be written in a single expression as

$$
\tilde{J}_{\beta}=\left|\left(\operatorname{det} B^{\prime}\right)^{(\beta n+2-\beta) / 2}(\operatorname{det} B)^{(\beta n+2-\beta) / 2}\right|^{2} .
$$


Also (25a $)$ and $(\underline{25 b})$ imply that $\operatorname{det} B^{\prime}=\operatorname{det} A^{\prime}$ and $\operatorname{det} B=\operatorname{det} A$; hence, $\tilde{J}_{\beta}$ can be expressed as

$$
\tilde{J}_{\beta}=\left|\operatorname{det}\left(A^{\prime} A\right)\right|^{(\beta n+2-\beta)} .
$$

Using $(\underline{22 a})$ and $(\underline{22 b})$ in the last expression we have

$$
\operatorname{det}\left(A^{\prime} A\right)=\frac{\operatorname{det}{\tilde{t^{\prime}}}_{c}^{\dagger} \operatorname{det}{\tilde{t_{c}}}_{c}^{\dagger}}{\operatorname{det}\left(I_{n}-\tilde{S}\langle\tilde{S}\rangle\right)^{2}} \text {. }
$$

Finally, from (20a $)$ and $(\underline{20 b})$ we can verify that $\left|\operatorname{det} \tilde{t}_{c}^{\prime}\right|=\left|\operatorname{det} \tilde{t}_{c}\right|$. Then $\tilde{J}_{\beta}$ is given by

$$
\tilde{J}_{\beta}=\left[\frac{\left|\operatorname{det}\left(I_{n}-\langle\tilde{S}\rangle\langle\tilde{S}\rangle^{\dagger}\right)\right|^{(\beta n+2-\beta) / 2}}{\left|\operatorname{det}\left(I_{n}-\tilde{S}\langle\tilde{S}\rangle^{\dagger}\right)\right|^{(\beta n+2-\beta)}}\right]^{2} .
$$

Equation (43), together with (39), is the main result of this work and can be interpreted as follows: if a non-unitary scattering matrix $\tilde{S}_{0}$ is uniformly distributed in the space of non-unitary scattering matrices, then another non-unitary scattering

matrix $\tilde{S}$, obtained from $\tilde{S}_{0}$ through the transformation given by (19), is distributed according to $\tilde{J}_{\beta}$ given by (43). In this sense, $\tilde{J}_{\beta}$ is the generalization of the Poisson kernel for non-unitary scattering matrices. We show in Appendix C that (39) together with (43) yields the Poisson kernel for unitary matrices. We remark that for unitary scattering matrices, the Poisson kernel has been originally obtained in the framework of maximum-entropy or Shannon information theory [3]. However, to the best of our knowledge, a derivation of the Poisson kernel for non-unitary scattering matrices from maximum-entropy arguments is not available.

\section{The one-channel case}

As an example, let us consider a $1 \times 1$ matrix $\tilde{S}$ which can be parametrized in polar form as

$$
\tilde{S}=\sqrt{R} \mathrm{e}^{\mathrm{i} \theta}
$$

where $R$ is the reflection coefficient and $\theta$ is the negative of twice the phase shift with $0 \leq R<1$ for sub-unitary matrices and $R>1$ for supra-unitary matrices.

In the sub-unitary case, a uniform distribution for $\tilde{S}$ means that it is distributed according to its invariant measure

$$
\mathrm{d} \mu_{\beta}(\tilde{S})=\mathrm{d} R \frac{\mathrm{d} \theta}{2 \pi} .
$$

A non-uniform distribution of $\tilde{S}$ is constructed from (45) as

$$
\mathrm{d} P^{(\beta)}(\tilde{S})=p^{(\beta)}(R, \theta) \mathrm{d} R \frac{\mathrm{d} \theta}{2 \pi} .
$$


For instance, if $\tilde{S}_{0}$ is the scattering matrix associated to chaotic cavities with losses in the absence of direct processes, $p_{0}^{(\beta)}\left(R_{0}, \theta_{0}\right)=p_{0}^{(\beta)}\left(R_{0}\right)$, where $p_{0}^{(\beta)}\left(R_{0}\right)$ is known [24, 26]. In the presence of direct processes, according to (39), with $\tilde{J}_{\beta}$ given by (43), we have

$$
\mathrm{d} R_{0} \frac{\mathrm{d} \theta_{0}}{2 \pi}=\left(\frac{1-|\langle\tilde{S}\rangle|^{2}}{\left|1-\tilde{S}\langle\tilde{S}\rangle^{*}\right|^{2}}\right)^{2} \mathrm{~d} R \frac{\mathrm{d} \theta}{2 \pi},
$$

which, multiplying by $p_{0}\left(R_{0}(R, \theta)\right)$, can be written as

$$
p_{0}\left(R_{0}(R, \theta)\right) \mathrm{d} R_{0} \frac{\mathrm{d} \theta_{0}}{2 \pi}=\left(\frac{1-|\langle\tilde{S}\rangle|^{2}}{\left|1-\tilde{S}\langle\tilde{S}\rangle^{*}\right|^{2}}\right)^{2} p_{0}\left(R_{0}(R, \theta)\right) \mathrm{d} R \frac{\mathrm{d} \theta}{2 \pi} .
$$

Comparing the right hand of side of Equations (46) and (48), we obtain that

$$
p^{(\beta)}(R, \theta)=\left(\frac{1-|\langle\tilde{S}\rangle|^{2}}{\left|1-\tilde{S}\langle\tilde{S}\rangle^{*}\right|^{2}}\right)^{2} p_{0}^{(\beta)}\left(R_{0}(R, \theta)\right) .
$$

This result, is in agreement with that of Ref. [31], where $p^{(1)}(R, \theta)$ was verified by comparing with experimental measurements in microwave chaotic cavities.

\section{Conclusions}

We have reduced the problem of scattering in the presence of direct processes to the case without such processes for $n \times n$ non-unitary scattering matrices. We use a transformation to map an ensemble of such matrices $\tilde{S}$ with $\langle\tilde{S}\rangle \neq 0$ to an ensemble of $\tilde{S}_{0}$ scattering matrices with $\left\langle\widetilde{S}_{0}\right\rangle=0$. In our theoretical framework, the direct processes are characterized by the average $\langle\tilde{S}\rangle$. Therefore, $\tilde{S}$ and $\tilde{S}_{0}$ describe a system in the presence and in the absence of direct processes, respectively. The Jacobian $\tilde{J}_{\beta}$ of the transformation turns out to be the square of the known Poisson kernel. In our analysis we consider general complex, symmetric, and self-dual scattering matrices, in analogy to the three basic symmetries in Dyson's scheme $\beta=2,1$, and 4 . We have found that if $\tilde{S}_{0}$ is uniformly distributed in the space of non-unitary scattering matrices, then $\tilde{S}$ obtained from $\tilde{S}_{0}$ through the transformation (19) is distributed according to $\tilde{J}_{\beta}$ given in (43). Our study extends known results for the probability distribution of non-unitary scattering matrices in the absence of direct process to the case in the presence of such processes. As a consequence, from the simplest case of a uniform distribution of $\tilde{S}$, it is possible to obtain more complex distributions, emerging from situations where prompt responses are relevant to the scattering problem, as has been illustrated in the one-channel case. 
Chaotic scattering with direct processes: A generalization of Poisson's kernel

\section{Appendix A. Jacobian $\tilde{J}_{\beta}$ for $\beta=4$}

The invariant measure for $\beta=4$ is given by (18). For self-dual $\delta M_{0}$ and $\delta M$ matrices, (24) implies that

$$
B^{\prime}=B^{R} \text {. }
$$

Using the standard notation for quaternions [2], the elements of $\delta M$ can be written as

$$
\delta M_{a b}=\sum_{\alpha=0}^{3} \delta M_{a b}^{(\alpha)} e_{\alpha}, \quad \alpha=0,1,2,3,
$$

where $\delta M_{a b}^{(\alpha)}$ is the projection of $\delta M_{a b}$ on the quaternion $e_{\alpha}$, where

$$
e_{0}=\left[\begin{array}{ll}
1 & 0 \\
0 & 1
\end{array}\right], e_{1}=\left[\begin{array}{cc}
i & 0 \\
0 & -i
\end{array}\right], e_{2}=\left[\begin{array}{cc}
0 & 1 \\
-1 & 0
\end{array}\right], e_{3}=\left[\begin{array}{ll}
0 & i \\
i & 0
\end{array}\right] \text {. }
$$

From (24), we have

$$
\delta M_{0 i j}^{(\alpha)}=\sum_{a, b} B_{i a}^{\prime} \delta M_{a b}^{(\alpha)} B_{b j} .
$$

As in Sect. 3.1, we consider first the special case of a diagonal matrix $B$ with

$$
\begin{aligned}
& B_{a b}=\lambda_{a} \delta_{a b}, \\
& B_{a b}^{\prime}=\lambda_{a}^{\prime} \delta_{a b} .
\end{aligned}
$$

Substituting Equations (A.5) and (A.6) into (A.4) we obtain

$$
\delta M_{0 i j}^{(\alpha)}=\lambda_{i}^{\prime} \lambda_{j} \delta M_{i j}^{(\alpha)} .
$$

Hence, the real and imaginary parts are related by

$$
\begin{aligned}
& \operatorname{Re}\left(\delta M_{0 i j}^{(\alpha)}\right)=\operatorname{Re}\left(\lambda_{i}^{\prime} \lambda_{j}\right) \operatorname{Re}\left(\delta M_{i j}^{(\alpha)}\right)-\operatorname{Im}\left(\lambda_{i}^{\prime} \lambda_{j}\right) \operatorname{Im}\left(\delta M_{i j}^{(\alpha)}\right) \\
& \operatorname{Im}\left(\delta M_{0_{i j}}^{(\alpha)}\right)=\operatorname{Im}\left(\lambda_{i}^{\prime} \lambda_{j}\right) \operatorname{Re}\left(\delta M_{i j}^{(\alpha)}\right)+\operatorname{Re}\left(\lambda_{i}^{\prime} \lambda_{j}\right) \operatorname{Im}\left(\delta M_{i j}^{(\alpha)}\right) .
\end{aligned}
$$

Therefore, we have

$$
\operatorname{Re}\left(\delta M_{0 i j}^{(\alpha)}\right) \operatorname{Im}\left(\delta M_{0 i j}^{(\alpha)}\right)=\left|\lambda_{i}^{\prime} \lambda_{j}\right|^{2} \operatorname{Re}\left(\delta M_{i j}^{(\alpha)}\right) \operatorname{Im}\left(M_{i j}^{(\alpha)}\right),
$$

where $\left|\lambda_{i}^{\prime} \lambda_{j}\right|^{2}$ is just the Jacobian of the transformation given by Equations (A.8) and (A.9). Thus, using (18) we obtain

$$
\mathrm{d} \mu_{4}\left(\tilde{S}_{0}\right)=\tilde{J}_{4} \mathrm{~d} \mu_{4}(\tilde{S}),
$$

where

$$
\begin{aligned}
\tilde{J}_{4} & =\prod_{\alpha=1}^{3} \prod_{i<j}\left|\lambda_{i}^{\prime} \lambda_{j}\right|^{2} \prod_{i \leq j}\left|\lambda_{i}^{\prime} \lambda_{j}\right|^{2}=\left|\frac{\left(\prod_{i \leq j} \lambda_{i}^{\prime} \lambda_{j}\right)^{4}}{\left(\prod_{i} \lambda_{i}^{\prime} \lambda_{i}\right)^{3}}\right|^{2} \\
& =\left|\frac{\left(\operatorname{det} B^{\prime}\right)^{2(n+1)}(\operatorname{det} B)^{2(n+1)}}{\left(\operatorname{det} B^{\prime}\right)^{3}(\operatorname{det} B)^{3}}\right|^{2}=\left|\left(\operatorname{det} B^{R}\right)^{2 n-1}(\operatorname{det} B)^{2 n-1}\right|^{2},
\end{aligned}
$$

which is the result presented in (40) for $\beta=4$.

As in Sect. 3.2, the general case of a non diagonal matrix $B$ can be reduced to a diagonal one by means of Equations ( $(\underline{31 a})$ and $(\underline{31 b})$ with $Q^{\prime}=O^{R}$ and $O^{\prime}=Q^{R}$. Thus, we have the same result for $\tilde{J}_{4}$. 
Chaotic scattering with direct processes: A generalization of Poisson's kernel

\section{Appendix B. Explicit calculation of Eq. (37)}

For $\beta=1, \lambda_{a}^{\prime}=\lambda_{a}$ and the products appearing in the right-hand side of Eq. (37) can be written explicitly as

$$
\begin{aligned}
& \prod_{a \leq b} \lambda_{a} \lambda_{b}=\prod_{a=1}^{n} \prod_{b=a}^{n} \lambda_{a} \lambda_{b}
\end{aligned}
$$

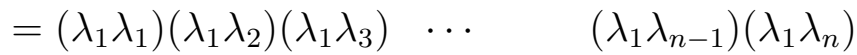

$$
\begin{aligned}
& \times \quad\left(\lambda_{2} \lambda_{2}\right)\left(\lambda_{2} \lambda_{3}\right) \quad \cdots \quad\left(\lambda_{2} \lambda_{n-1}\right)\left(\lambda_{2} \lambda_{n}\right) \\
& \times \quad\left(\begin{array}{lll}
\left.\lambda_{3} \lambda_{3}\right) & \cdots & \left(\lambda_{3} \lambda_{n-1}\right)\left(\lambda_{3} \lambda_{n}\right)
\end{array}\right. \\
& \times \quad\left(\lambda_{n-1} \lambda_{n-1}\right)\left(\lambda_{n-1} \lambda_{n}\right) \\
& \times \quad\left(\lambda_{n} \lambda_{n}\right)
\end{aligned}
$$

We can see that $\lambda_{1}$ appears $n+1$ times in the first line only, $\lambda_{2}$ once in the first line and $n$ times in the second line, $\lambda_{3}$ appears once in the first and second lines and $n-1$ times in the third line, etc. Then each $\lambda_{a}$ appears $n+1$ times in total and the product can be written as

$$
\prod_{a \leq b} \lambda_{a} \lambda_{b}=\prod_{a=1}^{n} \lambda_{a}^{n+1}=\left(\prod_{a=1}^{n} \lambda_{a}\right)^{n+1}=(\operatorname{det} B)^{n+1}
$$

For $\beta=2$, we have

$$
\begin{aligned}
\prod_{a, b=1}^{n} \lambda_{a}^{\prime} \lambda_{b} & =\prod_{a=1}^{n}\left(\prod_{b=1}^{n} \lambda_{a}^{\prime} \lambda_{b}\right)=\prod_{a=1}^{n}\left(\lambda_{a}^{\prime n} \prod_{b=1}^{n} \lambda_{b}\right) \\
& =\prod_{a=1}^{n} \lambda_{a}^{\prime n} \prod_{b=1}^{n} \lambda_{b}^{n}=\left(\prod_{a=1}^{n} \lambda_{a}^{\prime}\right)^{n}\left(\prod_{b=1}^{n} \lambda_{b}\right)^{n} \\
& =\operatorname{det} B^{\prime n} \operatorname{det} B^{n} .
\end{aligned}
$$

We can summarize the result for any $\beta$ as

$$
\prod_{\{a, b\}}^{n} \lambda_{a}^{\prime} \lambda_{b}=\operatorname{det} B^{\prime(\beta n+2-\beta) / 2} \operatorname{det} B^{(\beta n+2-\beta) / 2},
$$

which is also valid for $\beta=4$ (See Appendix A).

\section{Appendix C. Reduction to the Poisson kernel for unitary matrices}

We show in this appendix that the original Poisson kernel can be obtained by restricting a non-unitary $\tilde{S}$ matrix to be unitary.

On one hand, we are concerned with unitary scattering matrices $S_{0}=U_{0} V_{0}$ and $S=U V$ related by (7). Their measures satisfy (9). Therefore, if the probability density distributions of $S_{0}$ and $S$ are $p_{0}\left(S_{0}\right)$ and $p(S)$, respectively, $p_{0}\left(S_{0}\right)$ and $p(S)$ are related by

$$
p(S)=p_{0}\left(S_{0}\right) J_{\beta}, \quad \text { or } \quad p_{0}\left(S_{0}\right)=\frac{p(S)}{J_{\beta}} .
$$


In particular, we assume that $p(S)=\delta\left(\tilde{S} \tilde{S}^{\dagger}-I_{n}\right)$, where $\tilde{S}=U \rho V$ is a non-unitary matrix which is a function of $S$. Then, $\tilde{S}_{0}=U_{0} \rho_{0} V_{0}$ is a function of $S_{0}$, related to $\tilde{S}$ via (19). This leads to $p_{0}\left(S_{0}\right)=\delta\left(\tilde{S}_{0} \tilde{S}_{0}^{\dagger}-I_{n}\right)$, where $\tilde{S}_{0}$ is a function of $S_{0}$. From (C.1) we have that

$$
\delta\left(\rho_{0}-I_{n}\right)=\frac{\delta\left(\rho-I_{n}\right)}{J_{\beta}} .
$$

On the other hand, consider non-unitary scattering matrices, starting with (39) with $\tilde{J}_{\beta}$ given by (43). Here, $\tilde{S}_{0}$ is uniformly distributed in the space of non-unitary scattering matrices. We restrict to the space of unitary scattering matrices, imposing unitarity on $\tilde{S}_{0}$ by multiplying (39) by $\delta\left(\rho_{0}-I_{n}\right)$. We obtain

$$
\delta\left(\rho_{0}-I_{n}\right) d \mu\left(\tilde{S}_{0}\right)=\delta\left(\rho_{0}-I_{n}\right) \tilde{J}_{\beta} d \mu(\tilde{S}) .
$$

Using (C.2), (C.3) yields

$$
\delta\left(\rho_{0}-I_{n}\right) d \mu\left(\rho_{0}\right) d \mu\left(S_{0}\right)=\frac{\delta\left(\rho-I_{n}\right)}{J_{\beta}} \tilde{J}_{\beta} d \mu(\rho) d \mu(S),
$$

where we have written $d \mu(\tilde{S})=d \mu(\rho) d \mu(S)$. On the left-hand side, the integral with respect to $\rho_{0}$ gives one, while the integral with respect to $\rho$ on the right-hand side evaluates to $\tilde{J}_{\beta}$ at $\rho=I_{n}$. This implies $\tilde{J}_{\beta}\left(\rho=I_{n}\right)=J_{\beta}^{2}($ compare (9) with (43)). Finally, we get

$$
d \mu\left(S_{0}\right)=\frac{J_{\beta}^{2}}{J_{\beta}} d \mu(S)=J_{\beta} d \mu(S),
$$

where $J_{\beta}$ is the Poisson kernel for unitary scattering matrices $S$.

\section{Acknowledgments}

We thank J. Flores and D. Sanders for carefully reading this manuscript. Also, we thank the DGAPA-UNAM, México for financial support, under project IN118805. VAG acknowledges financial support from the Ministerio de Educación y Ciencia, Spain, through the Ramón y Cajal Program and the project FIS 2006-08-532, as well as the hospitality during his visit to the ICF-UNAM and UAM-Iztapalapa. MMM thanks BIFI and the Departamento de Física Teórica of the Universidad de Zaragoza for kind hospitality.

[1] Porter C E 1965 Statistical theories of spectra: fluctuations (New York: Academic Press)

[2] Metha M L 2004 Random Matrices (Amsterdam: Academic Press) 3rd ed.

[3] Mello P A and Kumar N 2004 Quantum Transport in Mesoscopic Systems. Complexity and statistical fluctuations (New York: Oxford University Press)

[4] Beenakker C W J 1997 Rev. Mod. Phys. 69731

[5] Alhassid Y 2000 Rev. Mod. Phys. 72895

[6] Dyson F J 1962 J. Math. Phys. 3140

[7] Mello P A, Pereyra P and Seligman T H 1985 Ann. Phys., NY 161254

[8] Friedman W A and Mello P A 1985 Ann. Phys., NY 161276

[9] Hua L K 1963 Harmonic Analysis of Functions of Several Complex Variables in the Classical Domains (L. Ebner and A. Korányi, Tr.) (Providence R I: Amer Math Soc) 
[10] Mello P A and Baranger H U 1999 Wave Random Media 9105

[11] Brouwer P W 1995 Phys. Rev. B 5116878

[12] Gopar V A and Mello P A 1995 Phys. Rev. B 5116917

[13] Bulgakov E N, Gopar V A, Mello P A, Rotter I 2006 Phys. Rev. B 73155302

[14] Kottos T and Smilansky U 2003 J. Phys. A: Math. Gen. 363501

[15] Fyodorov Y V and Sommers H-J 1997 J. Math. Phys. 381918

[16] Fyodorov Y V, Kottos T and Stöckmann H-J (Eds.) 2005 Trends in quantum chaotic scattering J. Phys. A: Math. Gen. $\mathbf{3 8}$ No. 49

[17] Martínez-Mares M, Akguc G and Méndez-Sánchez R A 2006 cond-mat/0610521

[18] Alt H, Bäcker A, Dembowski C, Gräf H-D, Hofferbert R, Rehfeld H and Richter A 1998 Phys. Rev. E $\mathbf{5 8} 1737$

[19] Barth M, Kuhl U and Stöckmann H-J 1999 Phys. Rev. Lett. 822026

[20] Hul O, Tymoshchuk O, Bauch S, Koch P M and Sirko L 2005 J. Phys. A: Math. Gen. 3810489

[21] Schaadt K and Kudrolli A 1999 Phys. Rev. E 60 R3479

[22] Morales A, Gutiérrez L and Flores J Am. J. Phys. 69517

[23] Beenakker C W J, Paasschens J C J, and Brouwer P W 1996 Phys. Rev. Lett. 761368

[24] Fyodorov Y V, Savin D V and Sommers H-J 2005 J. Phys. A: Math. Gen. 3810731

[25] Kuhl U, Stöckmann H-J and Weaver R 2005 J. Phys. A: Math. Gen. 3810433

[26] Martínez-Mares M and Méndez-Sánchez R A 2005 J. Phys. A: Math. Gen. 3810873

[27] Hemmady S, Hart J, Zheng X, Antonsen T M Jr., Ott E, Anlage S M 2006 cond-mat/0606650

[28] Martínez-Mares M and Mello P A 2005 Phys. Rev. E 72026224

[29] Domínguez-Rocha V, Zagoya C, and Martínez-Mares M, arXiv:0707.3841v1

[30] Báez G, Martínez-Mares M, and Méndez-Sánchez R A, arXiv:0710.2223

[31] Kuhl U, Martínez-Mares M, Méndez-Sánchez R A and Stöckmann H J 2005 Phys. Rev. Lett. 94 144101

[32] Méndez-Sánchez R A, Kuhl U, Barth M, Lewenkopf C H and Stöckmann H-J 2003 Phys. Rev. Lett. 91174102

[33] Brouwer P W and Beenakker C W J 1997 Phys. Rev. B 554695

[34] Kogan E, Mello P A and Liqun He 2000 Phys. Rev. E 61 R17 